\title{
REALTIME MONITORING OF CARDIAC PATIENTS AT DISTANCE USING TARANG COMMUNICATION
}

Christo Ananth $^{\# 1}$, S.Shafiqa Shalaysha ${ }^{\# 2}$, M.Vaishnavi ${ }^{\# 3}$, J.Sasi Rabiyathul Sabena $^{\# 4}$, A.P.L.Sangeetha ${ }^{\# 5}$, M.Santhi ${ }^{\# 6}$.

\#1 Assistant Professor, Department of ECE, Francis Xavier Engineering College, Tirunelveli

\#2, \#3,\#4,\#5,\#6 U.G.Scholar, Department of ECE, Francis Xavier Engineering College, Tirunelveli

\section{ABSTRACT}

Nowadays heart attack patients are increasing day by day."Though it is tough to save the heart attack patients, we can increase the statistics of saving the life of patients \& the life of others whom they are responsible for. The main design of this project is to track the heart attack of patients who are suffering from any attacks during driving and send them a medical need \& thereby to stop the vehicle to ensure that the persons along them are safe from accident. Here, an eye blinking sensor is used to sense the blinking of the eye. $\mathrm{spO}_{2}$ sensor checks the pulse rate of the patient. Both are connected to micro controller.

If eye blinking gets stopped then the signal is sent to the controller to make an alarm through the buffer. If spO $\mathrm{O}_{2}$ sensor senses a variation in pulse or low oxygen content in blood, it may results in heart failure and therefore the controller stops the motor of the vehicle. Then Tarang F4 transmitter is used to send the vehicle number \& the mobile number of the patient to a nearest medical station within $25 \mathrm{~km}$ for medical aid. The pulse rate monitored via LCD.The Tarang F4 receiver receives the signal and passes through controller and the number gets displayed in the LCD screen and an alarm is produced through a buzzer as soon the signal is received.

Key words: Eye Blink sensor, $\mathrm{spO}_{2}$ sensor, LCD screen, Tarang Transmitter, receiver

Corresponding Author: Christo Ananth

\section{INTRODUCTION}

Real time monitoring of cardiac patients are used to protect the heart patients, including alert systems and the vehicle onboard controllers with components that communicate with each other. Here we implement a cardiac patient monitoring system in which at the first level choose the Tarang F4 for the communication which has more coverage area than the Zigbee, for the communication between the vehicle and Hospital. Hence here we design a system which can be used by the vehicle and the Hospital which can communicate each other. We design an automated communication between the patient to Hospital. [1] presented a system which describes the ECG Monitoring using Zigbee. This paper presents the development of a system for wireless ECG monitoring and alarm using ZigBee. The system is intended for home use by patients that are not in a critical condition but need to be constant or periodically monitored by clinicians or family. Patient monitoring is the 
cornerstone of proper medical care. It provides clinicians the much needed information about a person's current health status, so that they can act accordingly if anything goes wrong. Nowadays, complex patient monitoring systems offer the possibility of continuously monitoring a multitude of biological signals, analyze them, interpret them and take the appropriate action; or alert clinicians if necessary. The device is built as a low-power, small-sized, low-cost solution suitable for monitoring elderly people at home or in a nursing facility without interfering with the daily activity of a patient. It should give sufficient information in real time, and make it available remotely. The intention is not to achieve perfect clinical accuracy but the device is able to detect anomalies in the measured data and it also has alerting features. Authorized observers (clinicians or family) can monitor at any moment the state of the patient through the internet. It is intended for home use by patients that are not in a critical condition but need to be constant or periodically monitored by clinicians or family. But however this method is not applicable for the patient in movement and there is no alarm or any alert indication.

[2] proposed a system describes the Blood Pressure Monitoring System For Heart Failure Patients. Patients who have cardiovascular system disorders can measure their weight, blood pressure, activity levels, and other vital signs in a real-time automated fashion. The patient's weight, blood pressure, activity levels, and other vital signs are measured in a real-time automated fashion. But however, this method is This method is suitable for the patient who are hospitalized. [3] describes the physiological parameters of the patient which are constantly monitored. In this paper, a role-based intelligent mobile care system is included with alert mechanism in chronic care system which includes patients, physicians, nurses, and health care providers. Each of the roles represents a person that uses a mobile device such as a mobile phone to communicate with the server setup in the care center such that he or she can go around without restrictions. For commercial mobile phones with Bluetooth communication capability attached to chronic patients, [3] developed physiological signal recognition algorithms that were implemented. It is thus possible to integrate several front-end mobile care devices with Bluetooth communication capability to extract patients' various physiological parameters [such as blood pressure, pulse, saturation of hemoglobin $\left(\mathrm{SpO}_{2}\right)$, and electrocardiogram (ECG)], to monitor multiple physiological signals without space limit, and to upload important or abnormal physiological information to healthcare center for storage and analysis or transmit the information to physicians and healthcare providers for further processing. However this method is not suitable for the driving patient.

Five topics are discussed in this project : Detecting the patient BPM and the Eye blinking status; Transmitting via Tarang F4 in case of abnormalities in patient; The patient status is displayed and indicated by Buzzer; The Hospital Unit receives the patient's mobile and the car number; The communication between the vehicle and the Hospital through Tarang F4. 


\section{MATERIALS AND METHODS}

\section{A. Car Unit}

Fig.1. shows The Transmitter Block Diagram of the Car Unit.

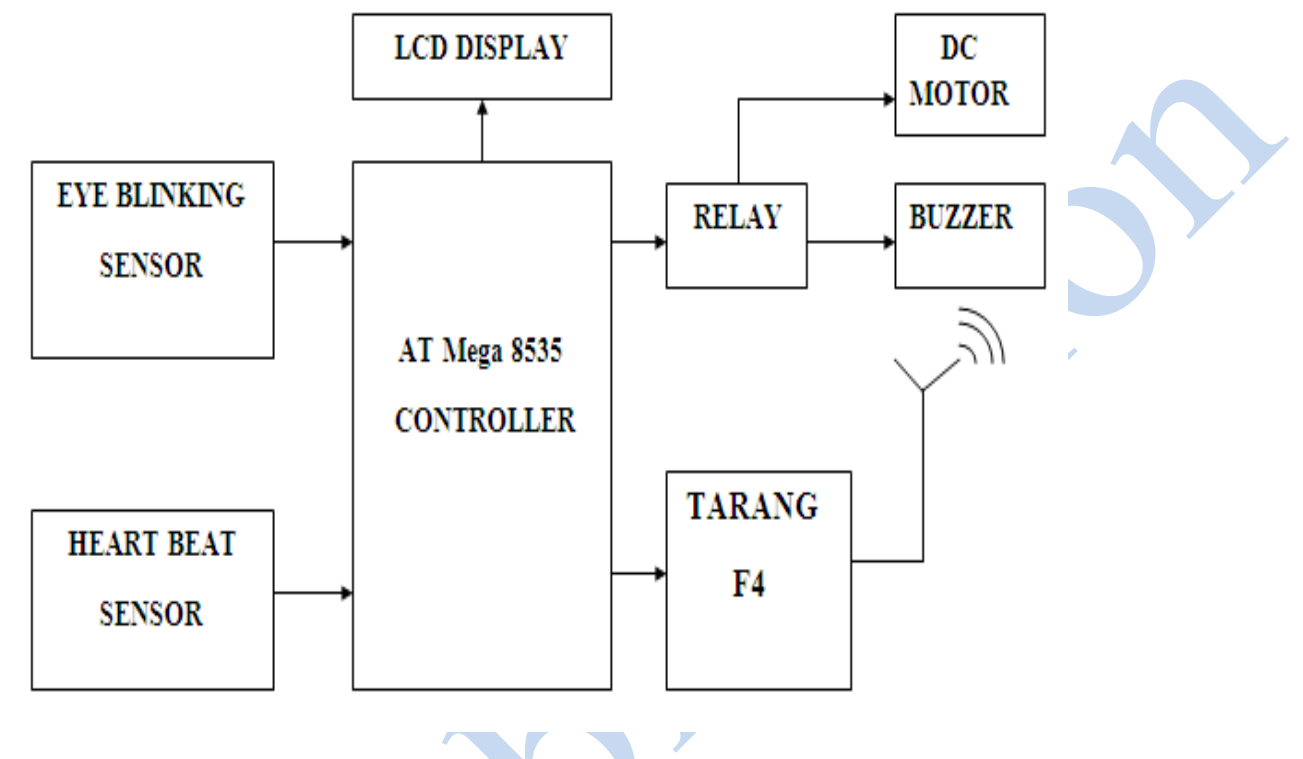

Fig 1: Block Diagram of Car Unit

The Car Unit consists of Microcontroller, Tarang F4, LCD Display, Relay, DC Motor, Eye Blinking (IR) Sensor, Heart Beat (IR) Sensor. . Here we use an eye blinking sensor to sense the eye blinking time, and a spo2 sensor to check the pulse rate of the patient. Both are connected to micro controller. If eye blinking gets stopped then the signal is sent to the controller to make an alarm through the buffer. If spo2 sensor senses a variation in pulse or low oxygen content in Blood it may results in heart failure then the controller stops the motor of the vehicle. Then Tarang F4 transmitter is used to send the vehicle no. \& the mobile no. of the patient to a nearest medical station within $25 \mathrm{~km}$ for medical aid. The pulse rate monitored via LCD. As soon as the signal received by the Tarang F4 it sends the data to the controller. The controller output is normally LOW, Therefore the Relay ON the Green LED. If the Controller output is High the Relay toggles to the Buzzer. The Controller displays the patient's Vehicle and the Mobile number .The car unit have AT Mega 8535 Microcontroller and the Hospital Unit have AT Mega 8 Microcontroller.

\section{B. Atmel ${ }^{\circledR}$ AVR ${ }^{\circledR}$ ATmega8}

The Atmel $®$ AVR $®$ ATmega8 is a low-power CMOS 8-bit microcontroller based on the AVR RISC architecture. By executing powerful instructions in a single clock cycle, the ATmega8 achieves throughputs approaching 1MIPS per $\mathrm{MHz}$, allowing the system designer to optimize power consumption versus processing speed. The Atmel® AVR ${ }^{\circledR}$ core combines a rich instruction set with 32 general purpose working registers. All the 32 registers are directly connected to the Arithmetic Logic Unit (ALU), allowing two independent registers to be 
accessed in one single instruction executed in one clock cycle. The resulting architecture is more code efficient while achieving throughputs up to ten times faster than conventional CISC microcontrollers.

\section{TARANG}

Tarang 2.4GHz Modules are suitable for adding wireless capability to any product with serial data interface. The modules require minimal power and provide reliable delivery of data between devices. The I/O interfaces provided with the Module help to directly fit into many industrial applications. The modules operate within the ISM $2.4 \mathrm{GHz}$ frequency with 802.15 .4 base band. A whip antenna is an antenna consisting of a single straight flexible wire or rod. The bottom end of the whip is connected to the radio receiver or transmitter. They are designed to be flexible so that they won't break off, and the name is derived from their whip-like motion when disturbed. Often whip antennas for portable radios are made of a series of interlocking telescoping metal tubes, so they can be retracted when not in use. Longer ones made for mounting on vehicles or structures are made of a flexible fiberglass rod surrounding a wire core, and can be up to $35 \mathrm{ft}$ (10 m) long.

\section{RESULTS AND DISCUSSION}

Fig.2. shows the LM358 Op-Amp. It consists of two independent, high gain, internally frequency compensated operational amplifiers which were designed specifically to operate from a single power supply over a wide range of voltages. Operation from split power supplies is also possible and the low power supply current drain is independent of the magnitude of the power supply voltage.

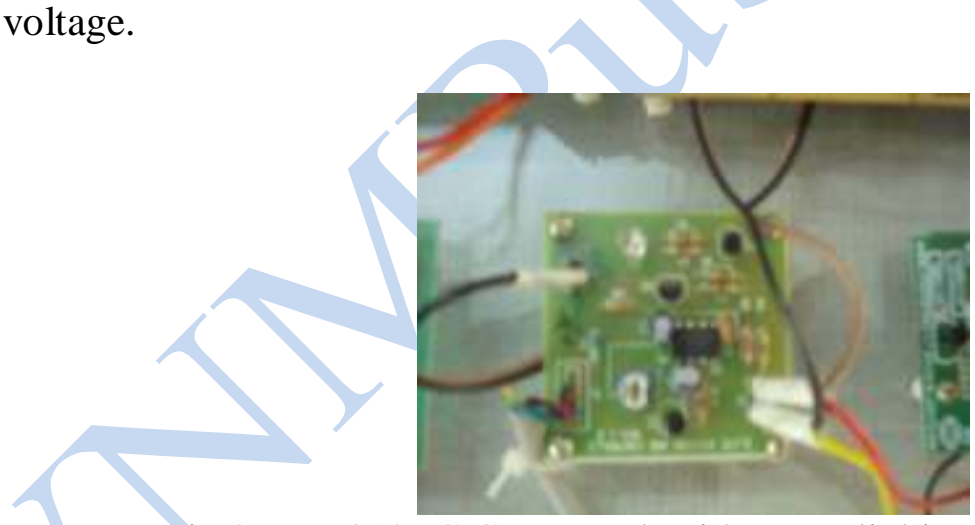

Fig.2: LM 358 IC Connected With Eye Blinking Sensor Circuit

Pulse Detector is a circuit which is used to measure the pulse at the finger tips. Fig.3. shows The Pulse Detection Sensor. A person's pulse can be detected by monitoring blood pressure changes within the human body. As the heart muscle contracts, blood pressure goes up and as it relaxes, blood pressure goes down. One clever method of monitoring blood pressure is to shine light through an appendage of the body and to measure the amount of light reaching a sensor. 


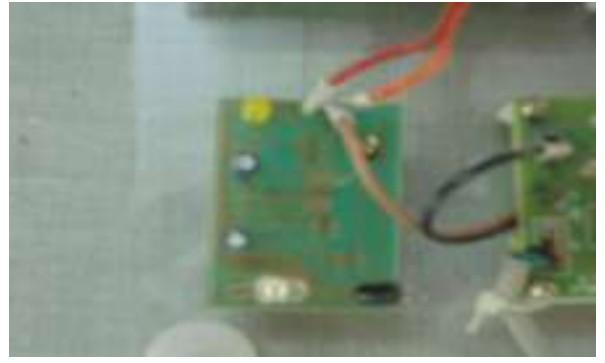

Fig.3: Pulse Detection sensor

The Car Unit is mounted near the driver's seat. If the driver is suffered by any attack or he is sleeping the controller warns the patient by a buzzer and the pulse rate, eye blinking time is displayed on the LCD screen. If the patient is normal only the pulse rate, eye blinking time is displayed on the LCD screen. If the patient is sleeping or pulse rate downs due to heart attack is indicated by a buzzer and the patient's record is transmitted to the nearby hospital by the Tarang F4. Then the motor is Turned OFF. Fig.4. shows the Top view of the Transmitter Car Unit consists of Eye and Pulse detecting sensor, TarangF4, Controller, LCD and Buzzer.

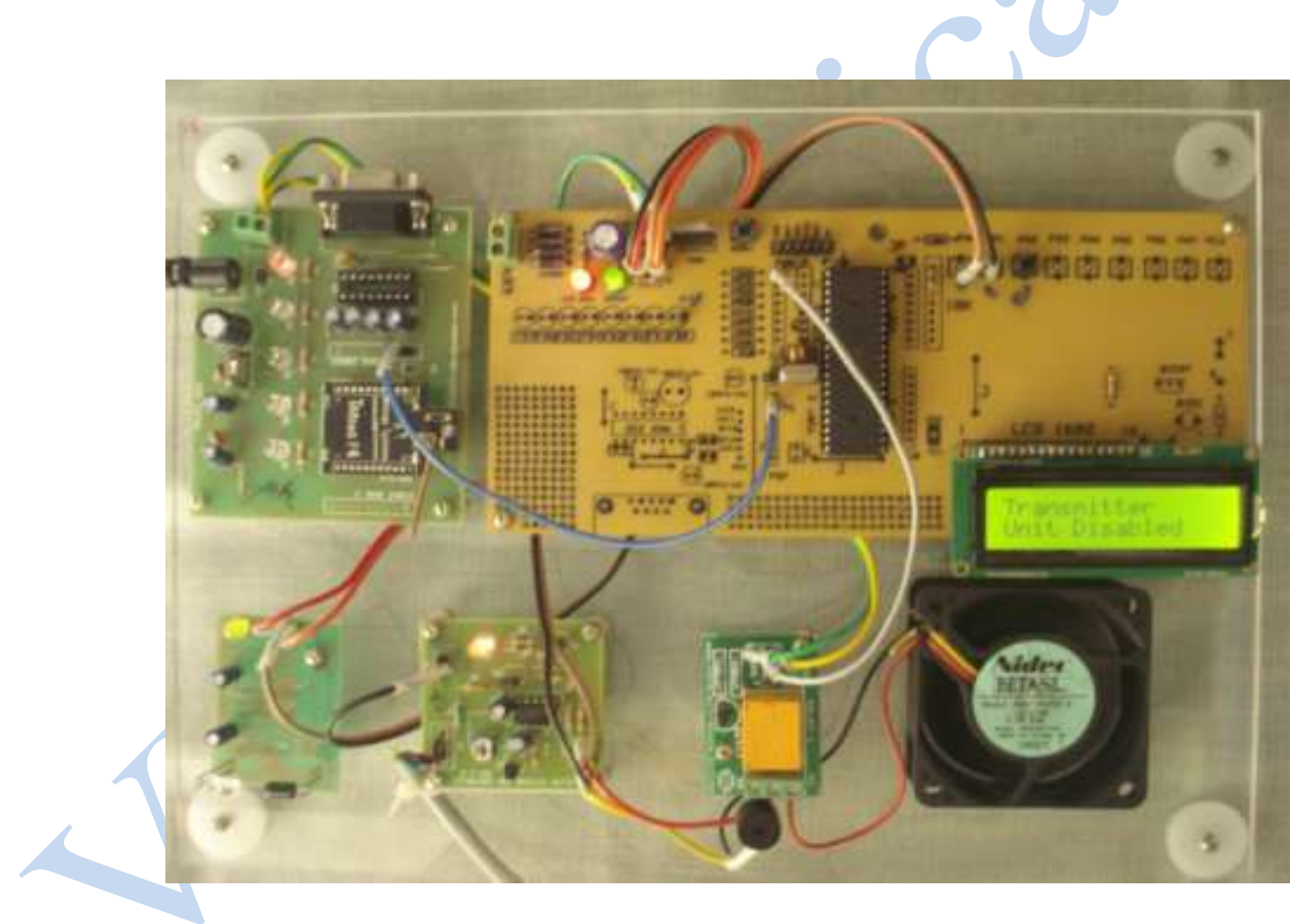

Fig 4: Top view of Transmitter Car Unit

In case of any critical situations, the car engine is turned off by using solenoid valve to drain the fuel. The patient's information is sent to the nearby hospital having Tarang module. The patient's car number \& mobile number is displayed on the LCD Screen of the Hospital Unit. and is indicated with a Buzzer. 


\section{CONCLUSION}

The main design of this project is to track the heart attack of patients who are suffering from any attacks during driving and send them a medical need $\&$ thereby to stop the vehicle to ensure that the persons along them are safe from accident. Here, an eye blinking sensor is used to sense the blinking of the eye. $\mathrm{spO}_{2}$ sensor checks the pulse rate of the patient. Both are connected to micro controller. If eye blinking gets stopped then the signal is sent to the controller to make an alarm through the buffer. If $\mathrm{spO}_{2}$ sensor senses a variation in pulse or low oxygen content in blood, it may results in heart failure and therefore the controller stops the motor of the vehicle. Then Tarang F4 transmitter is used to send the vehicle number \& the mobile number of the patient to a nearest medical station within $25 \mathrm{~km}$ for medical aid. The pulse rate monitored via LCD .The Tarang F4 receiver receives the signal and passes through controller and the number gets displayed in the LCD screen and an alarm is produced through a buzzer as soon the signal is received.This project is very useful for useful for heart patients and health Care Centres.

\section{REFERENCES}

[1] Apostu, O.; Hagiu, B.; Pasca, S., "Wireless ECG monitoring and alarm system using ZigBee," Advanced Topics in Electrical Engineering (ATEE), 2011 7th International Symposium on , vol., no., pp.1,4, 12-14 May 2013

[2] Myung-kyung Suh; Evangelista, L.S.; Chen, V.; Wen-Sao Hong; Macbeth, J.; Nahapetian, A.; Figueras, F.; Sarrafzadeh, M, 'WANDA B.: Weight and activity with blood pressure monitoring system for heart failure patients', IEEE International Symposium on a, vol., no., pp.1,6, 14-17 June 2012

[3] Ren-Guey Lee; Yih-Chien Chen; Chun-Chieh Hsiao; Chwan-Lu Tseng, 'A Mobile Care System With Alert Mechanism,' Information Technology in Biomedicine, IEEE Transactions on , vol.11, no.5, pp.507,517, Sept. 2011

[3] Mahananto, F.; Igasaki, T.; Murayama,(2013), N., 'Cardiac arrhythmia detection using combination of heart rate variability analyses and PUCK analysis,' Engineering in Medicine and Biology Society (EMBC), 2013 35th Annual International Conference of the IEEE , vol., no., pp.1696,1699, 3-7 July 2011

[4] Jain, N.P.; Jain, P.N.; Agarkar, T.P.,(2012), "An embedded, GSM based, multiparameter, realtime patient monitoring system and control - An implementation for ICU patients," Information and Communication Technologies (WICT), 2012 World Congress on , vol., no., pp.987,992, Oct. 30 2012-Nov. 22012

[5] Wei Lin,(2011), "Real time monitoring of electrocardiogram through IEEE802.15.4 network," Emerging Technologies for a Smarter World (CEWIT), 2011 8th International Conference \& Expo on , vol., no., pp.1,6, 2-3 Nov. 2010 